\title{
Study on the meshing force of misaligned gear coupling
}

\author{
Yunbo $\mathrm{Hu}^{1, \mathrm{a}}$, Huibin Wang ${ }^{1}$, Yuanqiang $\mathrm{Tan}^{2, \mathrm{~b}}$, Likuan Jiang ${ }^{3}$ and \\ Shengqiang Jiang ${ }^{3}$ \\ ${ }^{1}$ AECC South Aviation Industry Limited Corporation, Zhuzhou 412002,China \\ ${ }^{2}$ Huaqiao University, Xiamen 361021, China \\ ${ }^{3}$ Xiangtan University, Xiangtan 411105,China \\ ahyb_cheery@163.com, btanyq@hqu.edu.cn
}

Keywords: gear coupling; misalignment; meshing force; finite element method

Abstract. Gear couplings are used to transmit torque, and theoretically all teethes transmitted the same amount of torque and there is no difference in meshing force for each tooth. In practice, due to manufacturing and mounting errors, not all teeth transmit the same amount of torque, and then resulting in an uneven load between teeth and an over loading condition may occur. In this paper, a theoretical model of meshing force of gear coupling in misalignment and the finite element method was used to calculate the meshing force and its variation with the amount of misalignment. The calculated results and the simulation results were compared, and the reasons for the discrepancy were also analyzed. The results in this paper can provide a basis for further corrections to the theoretical model of meshing force of gear coupling in misalignment, and it may take a reference value for engineering applications.

\section{Introduction}

Gear coupling is a kind of mechanical components used to link the two rotating parts to transmit torque, and it was widely used in mechanical transmission system. Theoretically, the loading on each gear teeth of the coupling is uniform, but in fact, due to the influence of manufacturing errors and assembly errors, the coupling two rotors will produce misalignment. In rotating machinery, rotor misalignment can cause vibration of the rotor system, accelerated bearing wear and other adverse effects, and the axis device can cause the stress of each tooth, bear alternating load in rotation, and then resulting in fretting wear and fatigue damage. In the rotor system, this state cannot be avoided. The fault caused by misalignment accounts for about $60 \%$ of the rotor system fault[1].

Domestic and foreign scholars have made a lot of research on the problem of the misalignment of the gear coupling. Based on theoretical and numerical analysis, Al-Hussain and Redmond[2] have been studied the dynamic response of the rotor system with the coupling of the gear coupling. Jing et al. [3] analyzed the nonlinear dynamic characteristics and stability of a multi-disk rotor system supported by a sliding bearing in the case of the diaphragm type elastic coupling misalignment. Zhou [4] and $\mathrm{Gu}[5]$ et al. established differential equation of bending torsional vibration coupling misalignment. And then, the dynamic characteristics and stability of the rotor system with coupling misalignment are studied systematically in the aspects of theoretical derivation, numerical simulation and experimental testing[6-8]. However, few researches were focus on the meshing force of gear coupling with misalignment.

In this paper, the finite element method will be to calculate the gear coupling under the condition of misalignment of gear meshing force and its variation with the misalignment, and the misalignment of gear coupling meshing force model calculation results were compared, and the reasons for the inconsistency were also analyzed. 


\section{Gear coupling with misalignment of meshing theory analysis and calculation}

\section{Force analysis of meshing gear coupling misalignment}

In order to facilitate the analysis, assuming that the tooth type coupling misalignment occurs in the $\mathrm{X}$ axis forward,as shown in Figure 2, the gear tooth number on the $\mathrm{X}$ axis is 1 , and the counter clockwise number is $2,3,4, \ldots, \mathrm{J}, \ldots, \mathrm{Z}$.

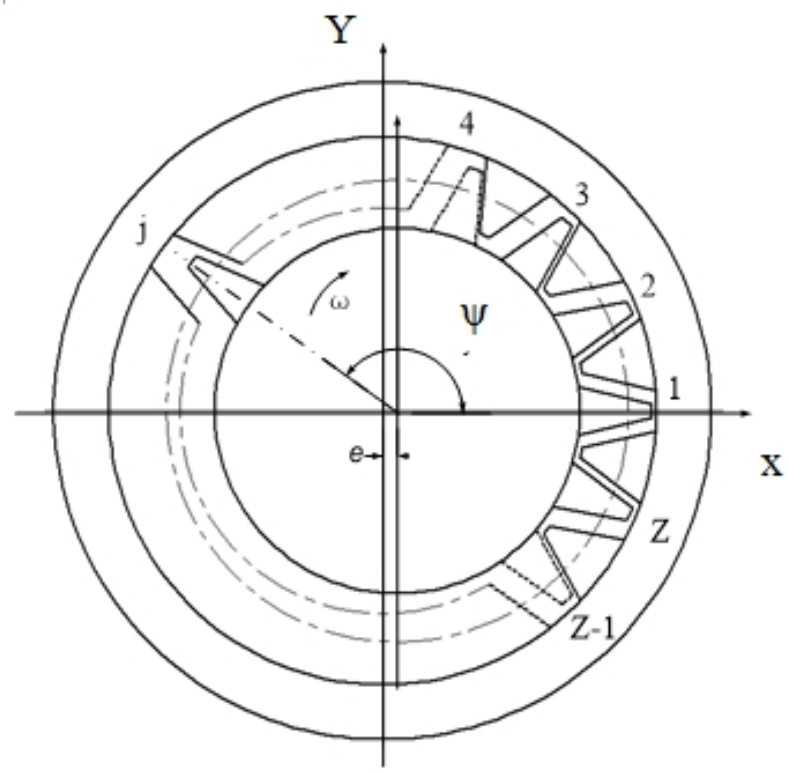

Fig. 1 Schematic of gear coupling with parallel misalignment in X-axis positive direction.

Thus, the angle between the teeth of the number $\mathrm{J}$ and the $\mathrm{X}$ axis positive is given as

$$
\varphi_{j}=2 \pi(j-1) / Z
$$

the equivalent mesh distance of each gear tooth can be described in the form

$$
L_{j}=L_{0}-e \cos \varphi_{j}
$$

where $L_{0}$ is the complete coupling tooth distance, and it is equal to dedendum.

The gear coupling through the interaction of internal and external teeth to transfer torque $\mathrm{T}$, thus

$$
\mathrm{T}=\sum_{\mathrm{j}=1}^{\mathrm{z}} \mathrm{T}_{\mathrm{j}}=\sum_{\mathrm{j}=1}^{\mathrm{z}}\left[\mathrm{F}_{\mathrm{tj}} \bullet \mathrm{R}_{\mathrm{j}}\right]=\sum_{\mathrm{j}=1}^{\mathrm{z}}\left[\mathrm{F}_{\mathrm{nj}} \cos \beta_{\mathrm{j}} \bullet\left(\mathrm{R}_{\mathrm{f}}+\mathrm{L}_{\mathrm{j}}\right)\right]
$$

where $R_{f}$ is the coupling tooth root radius, $R_{j}$ is the circular radius, $\beta_{j}$ is the engagement circle pressure angle, and $T_{j}$ is torque of the transmission of the first $\mathrm{j}$ tooth of the coupling.

And then, the meshing force (normal force) $F_{n j}$ can be described in the following form

$$
F_{n j}=\lambda_{j} \bullet K_{j}=\phi \frac{L_{j}}{\cos \beta_{j}} \bullet K_{j}
$$

where $\lambda_{j}$ is the deformation along the direction of the gear meshing force, $\varphi$ is the twist angle displacement of the force deformation of gear teeth.

The transfer torque can be expressed in another form by the formula (1), (2), thus

$$
T=\sum_{j=1}^{z}\left[\phi L_{j} K_{j} \bullet\left(R_{f}+L_{j}\right)\right]
$$

and then the twist angle displacement $\varphi$ can be obtained by the following form

$$
\varphi=\frac{T}{\sum_{j=1}^{z}\left[L_{j} K_{j}\left(R_{f}+L_{j}\right)\right]}
$$

therefore, the single tooth meshing force(normal) $F_{n j}$ can be derived as follows 


$$
F_{n j}=\frac{\phi L_{j} K_{j}}{\cos \beta_{j}}=\frac{T L_{j} K_{j}}{\cos \beta_{j} \bullet \sum_{j=1}^{z}\left[L_{j} K_{j}\left(R_{f}+L_{j}\right)\right]}
$$

\section{Calculation of meshing force with misaligned gear coupling}

In this paper, the structural parameters of the gear coupling and the related calculation parameters are shown in Table 1.

Table 1 The Parameters of gear coupling

\begin{tabular}{llllllll}
\hline $\begin{array}{l}\text { Modulus } \\
{[\mathrm{mm}]}\end{array}$ & $\begin{array}{l}\text { Tooth } \\
\text { number }\end{array}$ & $\begin{array}{l}\text { Pressure } \\
\left.\text { angle }^{\circ}\right]\end{array}$ & $\begin{array}{l}\text { Tooth } \\
\text { width[mm] }\end{array}$ & $\begin{array}{l}\text { Diameter } \\
\text { of } \\
\text { reference } \\
\text { circle[mm] }\end{array}$ & $\begin{array}{l}\text { Diameter } \\
\text { of tip } \\
\text { circle[mm }]\end{array}$ & $\begin{array}{l}\text { Young's } \\
\text { modulus } \\
{[\mathrm{GPa}]}\end{array}$ & $\begin{array}{l}\text { Poisson's } \\
\text { ratio }\end{array}$ \\
\hline 3 & 25 & 30 & 35 & 75 & 78 & 209 & 0.269 \\
\hline
\end{tabular}

Assuming the transfer torque of gear coupling is $1000 \mathrm{Nm}$, and the misalignment in the positive direction of axis $X$ is $0 \mathrm{~mm}, 0.02 \mathrm{~mm}, 0.04 \mathrm{~mm}, 0.06 \mathrm{~mm}$, respectively. According to the formula (7), the meshing force distribution of each teeth can be calculated and shown as in Figure 2.

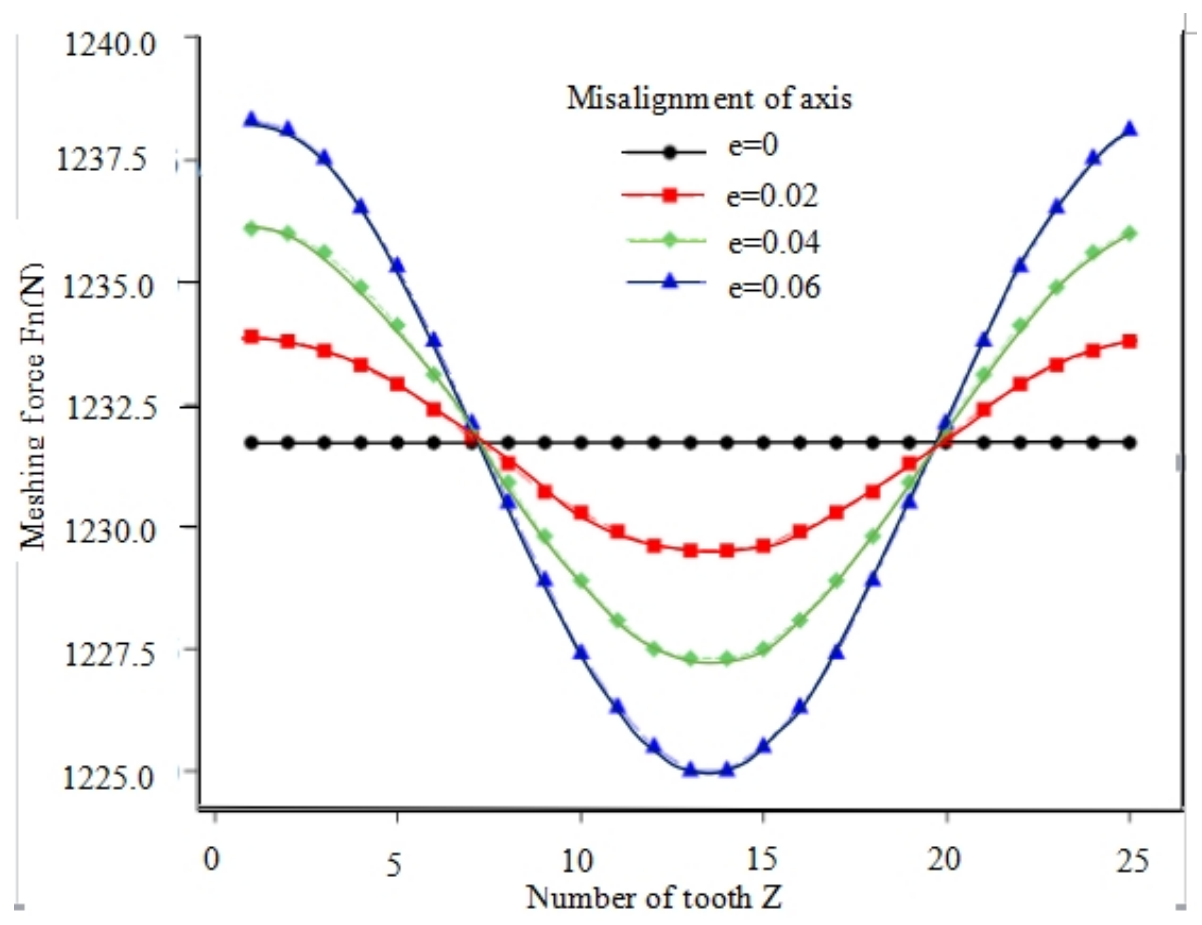

Fig. 2 Meshing force versus the number of tooth with different misalignment (theoretical model) 


\section{The meshing force of misaligned gear coupling by finite element analysis}

\section{Establishment of finite element model}

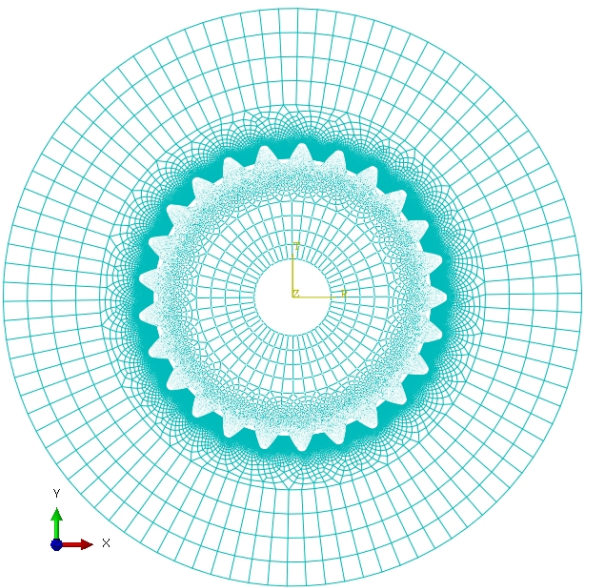

Fig. 3 FEM model of gear coupling

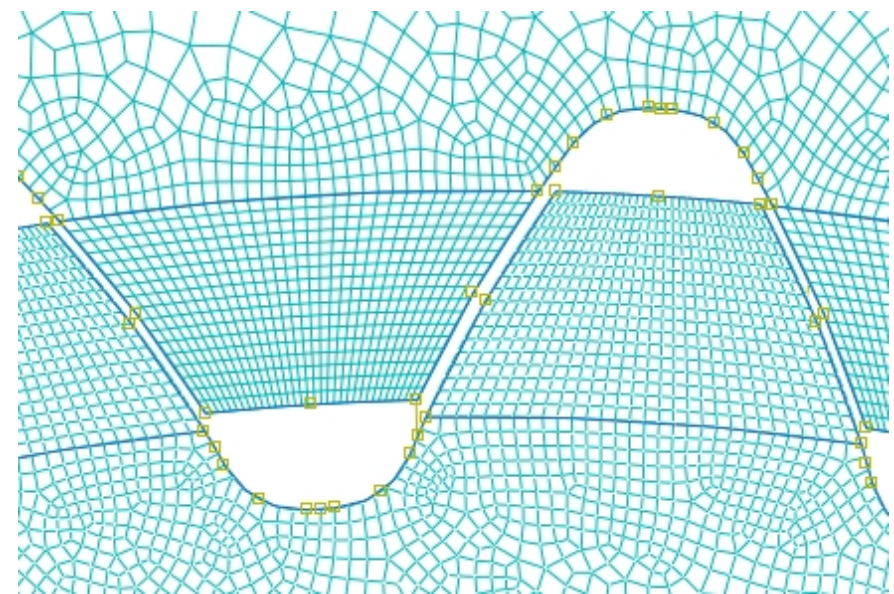

Fig. 4 Teeth mesh refinement and the contact settings

The assembly model of gear coupling is established with the finite element software ABAQUS, and the geometrical modeling parameters and material parameters are shown in Table 1. In this model, a two-dimensional finite element model was used to analysis, and the unit type production is a plane with four node quadrilateral plane strain bilinear intetgrated two-dimensional solid element CPE4R, it was shown as in Figure 3.

In order to ensure the accuracy of calculation, the gear tooth was segmented for preforming the local mesh refinement processing, and the contact between internal and external teeth is set to face to face contact mode, as shown in Figure 4. The inner ring of the inner coupling and the reference point will fully constraints, the outer coupling of an outreach and another reference point is combined with the applied counter clockwise torque, thus the set of boundary conditions was completed. The finite element model is divided into 56063 nodes and 53774 elements.

\section{Results of finite element analysis}

In order to compare with the theoretical calculation results, the finite element method is used to simulate the calculation of the transmission torque of the gear coupling in $1000 \mathrm{Nm}$, the misalignment in positive direction of $X$ axis was also set as $0 \mathrm{~mm}, 0.02 \mathrm{~mm}, 0.04 \mathrm{~mm}$ and $0.06 \mathrm{~mm}$ respectively, and the stress distribution under four conditions was shown in Figure 5 and the change of meshing force was shown in Figure 6.

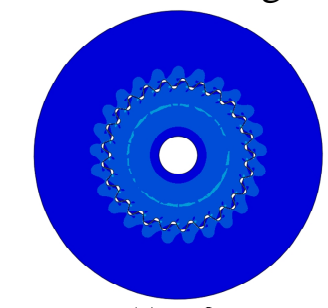

(a) $\mathrm{e}=0$

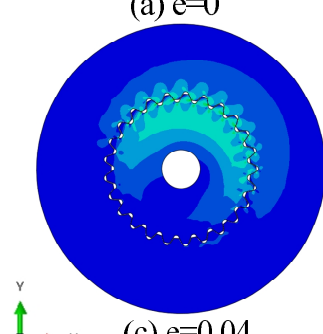

(c) $\mathrm{e}=0.04$

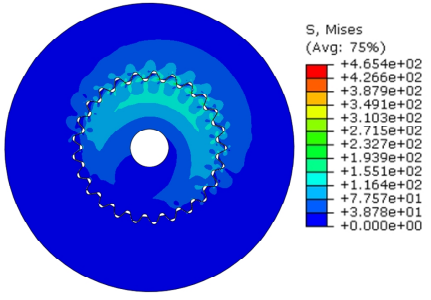

(b) $\mathrm{e}=0.02$

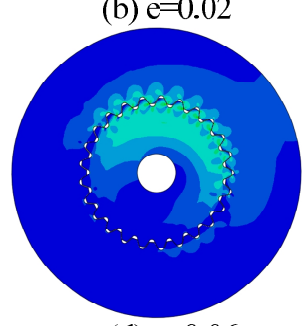

(d) $\mathrm{e}=0.06$

Fig.5 Stress variation of gear coupling

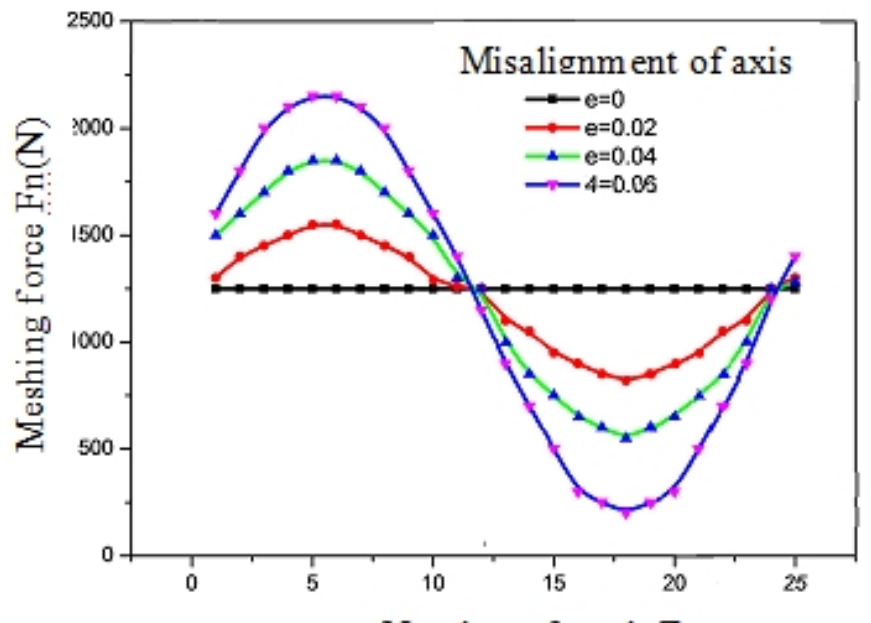

Number of tooth $Z$

Fig. 6 Meshing forces versus the number of tooh with different parallel misalignment 


\section{Discussion and analysis}

In this paper, a misaligned gear coupling based on meshing force model of gear coupling under the condition of misalignment of each meshing forces was calculated, and the results were shown in Figure 2. It can be seen that the misalignment have obvious influence on meshing forces. When the misalignment is $0 \mathrm{~mm}$ (there is no misalignment in the gear meshing force), the meshing force of each teeth will be equal and it was distributed in a horizontal line. When there is misalignment, the gear meshing force will not be equal, the maximum meshing force was occurred in the opposite direction from the misalignment of the teeth, and then it decreased gradually to both sides of the tooth, and the minimum meshing force was in direction of misalignment of the teeth. With the increase of the misalignment, the distribution of meshing forces is not uniform and the numerical difference is bigger.

In the case of misalignment, a certain torque is applied to the coupling, and the contact and meshing of the teeth are not carried out at the same time. While the theoretical model of the meshing forces of the gear coupling proposed in the literature [9]suggests that the meshing of the teeth is carried out at the same time and the twist angular displacements produced by the deformation of the teeth are equal, which leads to the model results (Figure2) with the calculated results of finite element model (Figure6) is different.

The engagement order of internal and external gear teeth is related to the tooth side clearance, as shown in Figure 8. When the coupling is complete, the tooth side gap of the coupling is $\eta_{0}$, when there is a misalignment, the tooth side gap of the gear tooth of the second $\mathrm{J}$ can be expressed by the following form

$$
\eta_{j}=\eta_{0}-e \cdot \cos \left(\varphi_{j}-\pi / 2+\beta\right)
$$

where $\eta_{0}$ is tooth side gap of fully to medium coupling, $\varphi_{j}$ is the angle between the tooth of $\mathrm{J}$ and the positive of $\mathrm{X}$ axis, $\beta$ is pressure angle.

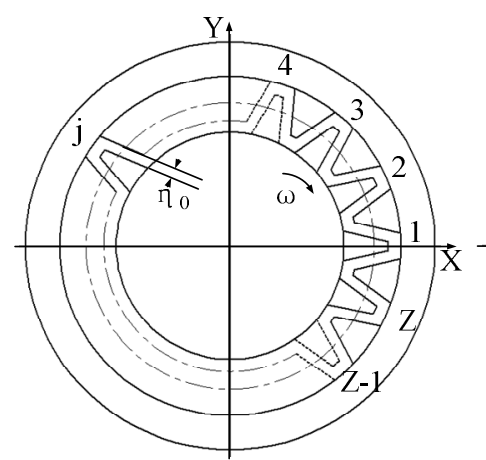

(a) completely alignment

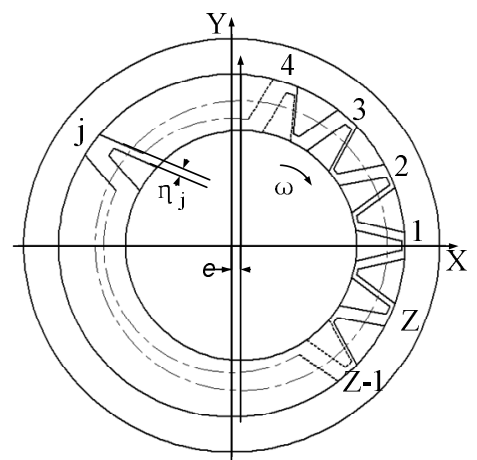

(b)misalignment

Fig. 7 The tooth backlash of gear coupling 


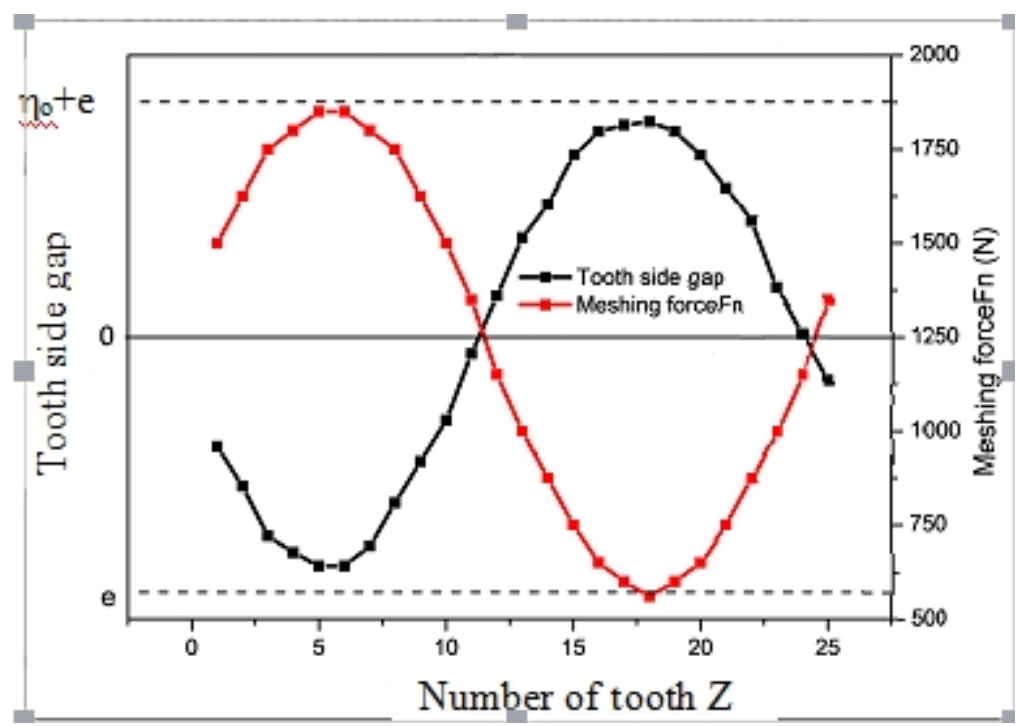

Fig.8 The relationship between tooth backlash and meshing force

Under ideal conditions, the tooth gap between the teeth is equal. When there is a misalignment, the gap is not equal. Under the action of torque, the tooth with the minimum side clearance will be first engagement. As the increasing of torque, the gear meshing backlash in accordance with the order from small to large, and the deformation and stress of the gear teeth are related to the tooth flank clearance and meshing order. The relationship between tooth backlash and meshing force was shown in Figure 8, it can be seen that the minimum backlash of tooth sustain the maximum meshing force, and the reverse is also true. When the external torque was equal to the torque generated by all meshing forces, it was reached in a equilibrium state.

From formula (8), it is clear to see that under the misaligned circumstances, the smallest backlash exists in gear teeth between which and the forward angle of $X$ axis the intersection angle is $(\pi / 2-\beta)$ and at the same time the gear teeth can bear the strongest mesh force. This result is in consistent with the finite element simulation result, which verifies the correctness of the finite element simulation result and also shows that there is deficiency in the theoretical model used to calculate the misaligned mesh force of gear coupling pointed out in the literature [9]. The result of this research can provide people a basis to further revise the theoretical model of misaligned mesh force of gear coupling and can be taken as a reference as well as used in engineering areas.

\section{Conclusions}

(1). Based on the misaligned gear coupling meshing force theory model, meshing force of the gear coupling and its variation under misaligned conditions are calculated in this paper. This paper elaborates the gear coupling meshing forces under same circumstances by using finite element method and its variation under misaligned conditions, which is compared with the conslusion made from the theoretical model. And the reason for the inconsistency is also analiyzed in this paper.

(2). In addition, this paper analyzed the variation of gear coupling backlash under misaligned conditions and the relation between meshing forces and backlash. The simulation results of finite element is explained and the deficiency of the theoretical model is also demenstrated by this paper.

\section{Acknowledgements}

This work was financially supported by Hunan provincial strategic emerging industries programs for science and technology development (No.2014GK1029). 


\section{References}

[1] $\mathrm{Li} \mathrm{M}, \mathrm{Yu} \mathrm{L}$. Analysis of the coupled lateral torsional vibration of a rotor-bearing system with a misaligned gear coupling[J]. Journal of Sound and Vibration, 2001, 243(2): 283-300.

[2] Al-Hussain K M, Redmond I. Dynamic response of two rotors co[1] Li M, Yu L. Analysis of the coupled lateral torsional vibration of a rotor-bearing system with a misaligned gear coupling[J]. Journal of Sound and Vibration, 2001, 243(2): 283-300.

nnected by rigid mechanical coupling with parallel misalignment[J]. Journal of Sound and Vibration, 2002, 249(3): 483-498.

[3] Wan Zhao, Jing JianPing, Meng Guang, Bai HuiYu. Nonlinear dynamic behavious and stability of a rotor-bearing system with flexible coupling misalignment[J]. Journal of Vibration and Shock,2012,31(24):20-25.

[4] Fu Bo, Zhou JianZhong, Peng Bing, An XueLi. Coupled lateral and torsional vibration properties of parallel misaligned fixed rigid couplings[J]. Journal of Huazhong University of Science and Technology(Nature Science Edition),2007,35(4):96-99.

[5] He ChengBing, Gu YuJiong, Yang Kun. Properties analysis on coupled lateral and torsional vibrations of the rotor connected by the gear coupling[J]. Journal of Mechanical Strength,2006,27(6):725-729.

[6] Liu ZhanSheng, Zhao Guang, Long Xin. Survey of the research on coupling with misalignment of rotary machinery[J]. Turbine Technology,2007,49(5):321-325.

[7] Zhao Guang, Liu ZhanSheng, Chen Feng, Wang YongLiang. Experiment research on misalignment and frequency-locking trouble of rotor-coupling-bearing-isolator system[J]. Journal of Dynamics and Control,2009,7(2):171-176.

[8] Zhao Guang, Guo JiaNan, Wang XiaoFang, Liu ZhanSheng. Dynamics of rotor-gear coupling-bearing system with misalignment[J]. Journal of Dalian University of Technology,2011,51(3):338-345.

[9] Liu ZhanSheng, Zhao Guang. Modeling research on radial force in gear coupling with parallel misalignment[C]//12th IFToMM World Congress, Besançon, France. 2007. 